\title{
Magnetic Hysteresis Models for Modelica
}

\author{
Johannes Ziske, Thomas Bödrich \\ Technische Universität Dresden, Institute of Electromechanical and Electronic Design \\ 01062 Dresden, Germany \\ Johannes.Ziske@tu-dresden.de \\ Thomas.Boedrich@tu-dresden.de
}

\begin{abstract}
Modelica models for transient simulation of magnetic hysteresis are currently being developed at Technische Universität Dresden. This paper gives an overview about the present state of the work. Two hysteresis models have been implemented so far in Modelica and are currently optimised and tested: the rather simple but efficient Tellinen model and the more complex and accurate Preisach model. Utilisation of the Tellinen model together with components of the Modelica.Magnetic.FluxTubes library is exemplarily shown with transient simulation of a threephase autotransformer. Additionally, an efficient implementation of the Preisach model is described and a first comparison between the Tellinen and the classical Preisach hystesis model is presented. It is planned to include the developed hysteresis models into the above-mentioned FluxTubes library after their further optimisation and validation with own measurements. These models will especially allow for the estimation of iron losses and for accurate computation of saturation behaviour during Modelica-based design of electromagnetic components and systems. This becomes increasingly important with the growing requirements regarding energy efficiency and mass power densities of such systems.
\end{abstract}

Keywords: magnetic hysteresis, lumped magnetic network; hysteresis model; Tellinen; Preisach; iron losses; Modelica.Magnetic.FluxTubes library

\section{Introduction}

The Modelica.Magnetic.FluxTubes library included in the Modelica Standard Library [1] is intended for rough design and system simulation of magnetic components and devices, e.g. actuators, motors, transformers or holding magnets [2, 3]. This library is based on the well-established concept of magnetic flux tubes, which enables modelling of magnetic fields with lumped networks [4].
At present, ferromagnetic hysteresis is not considered in the above-mentioned library. However, the prediction of losses due to static (ferromagnetic) and dynamic (eddy current) hysteresis becomes more and more important during the design of electromagnetic components. This is due to the increasing demands on energy efficiency of electromagnetic systems and due to increasing power densities of those systems. Prominent examples for this engineering trend are electromobility and more electric aircraft, where the necessity of high mass power densities and loss power minimisation are obvious.

In general, the reliable prediction of hysteresisrelated losses with lumped magnetic network models is difficult and demanding and has been a topic of research for a long time. Simplified empirical equations for loss calculation, e.g. the well-known Steinmetz formula [5] are based on time-harmonic flux densities of known magnitude and frequency [6]. The delayed penetration of magnetic fields into bulk and laminated ferromagnetic materials can be approximated in lumped magnetic networks with Cauer circuits [7].

Transient simulation of magnetic hysteresis in lumped magnetic network models is possible with dedicated hysteresis models. Well-known such models are for example the phenomenological one published by Preisach in 1935 [8], the physical model developed by Jiles and Atherton [9] or the comparatively simple model developed by Tellinen [10]. Those models are currently analysed at Technische Universität Dresden, and selected hysteresis models are implemented in Modelica for inclusion into the Modelica.Magnetic.FluxTubes library.

The Tellinen hysteresis model and the Preisach model have been implemented and are currently tested and optimised. Theory and Modelica implementation of these two models and their utilisation in components of the Modelica.Magnetic.FluxTubes library will be presented in the following sections. It must be noted that this is a report about work in progress rather than a final presentation of the projected Modelica.Magnetic.FluxTubes library extension. Both 
implemented hysteresis models are still subject to optimisation and validation, e.g. with measurements.

\section{The Tellinen Hysteresis Model}

\section{$2.1 \quad$ Theory}

The hysteresis model developed by Tellinen is thoroughly described in [10]. The big advantage of this model is its simplicity. Thus, it is well suited for fast simulations when used in lumped magnetic network models. It works without information about the history of the magnetic field strength $H$ in ferromagnetic components and can completely be configured with the limiting increasing and decreasing branches $\lambda_{\mathrm{i}}(H)$ and $\lambda_{\mathrm{d}}(H)$, respectively, of the limiting hysteresis loop of a ferromagnetic material (Figure 1).

a)

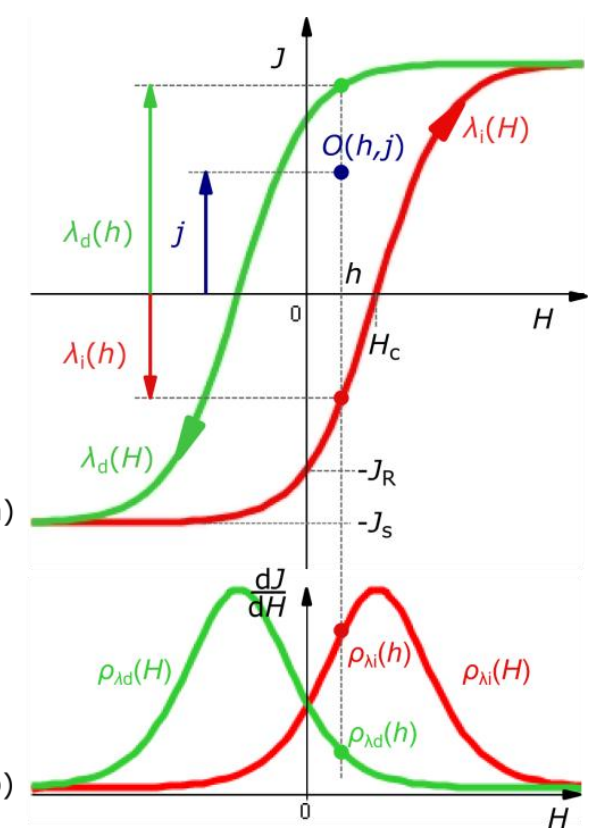

Figure 1: Limiting increasing and decreasing branch $\lambda_{\mathrm{i}}(H)$ and $\lambda_{\mathrm{d}}(H)$, respectively, of a hysteresis loop with magnetic polarization $J$ and magnetic field strength $H$ (a) and corresponding slope functions $\rho_{\lambda_{\mathrm{i}}}(H)$ and $\rho_{\lambda \mathrm{d}}(H)(\mathrm{b})$.

Together with the corresponding slope functions $\rho_{\lambda_{\mathrm{i}}}(H)$ and $\rho_{\lambda_{\mathrm{d}}}(H)$ the actual slope $\rho_{\mathrm{j}}$ at the operating point $O(h, j)$ can be determined as

$\rho_{j}=\frac{d j}{d H}= \begin{cases}\frac{\lambda_{d}(h)-j}{\lambda_{d}(h)-\lambda_{i}(h)} \cdot \rho_{\lambda i}(h) & \text { for } d H>0 \\ \frac{j-\lambda_{i}(h)}{\lambda_{d}(h)-\lambda_{i}(h)} \cdot \rho_{\lambda i}(h) & \text { for } d H<0 \\ 0 & \text { else. }\end{cases}$
Thus, the time-based slope of $j$ can be easily computed at every integration step to

$$
\frac{d j}{d t}=\frac{d j}{d H} \cdot \frac{d H}{d t}=\rho_{j} \frac{d H}{d t} .
$$

Hence the slope of the magnetic flux density $\mathrm{d} b / \mathrm{d} t$ of

$$
\frac{\mathrm{d} b}{d t}=\left(\rho_{j}+\mu_{0}\right) \cdot \frac{d H}{d t}
$$

$\mu 0$ is the slope $\mathrm{d} b / \mathrm{d} h$ of the limiting hysteresis loops within the saturation region.

\subsection{Implementation in Modelica}

The Tellinen model described above was integrated into a reluctance element of the Modelica.Magnetic.FluxTubes library, and can thus similarly be used in electromagnetic network models (in [2] the magnetic library is explained in detail). The reluctance model can be configured with the cross section and the length of a ferromagnetic core and the limiting hysteresis loop of the core material. On the one hand hysteresis loops can be defined by the hyperbolic tangent function and definition of the three parameters $J_{\mathrm{S}}$ (saturation polarization), $J_{\mathrm{R}}$ (remanence) and $H_{\mathrm{C}}$ (coercivity) (see Figure 1a). On the other hand table data can be used to define the increasing and decreasing hysteresis branches. Thus, almost arbitrary hysteresis loops can easily be implemented and also easily be derived from measurements. In addition a small experimental library was built using exemplary table data of some common ferromagnetic materials (Figure 2).

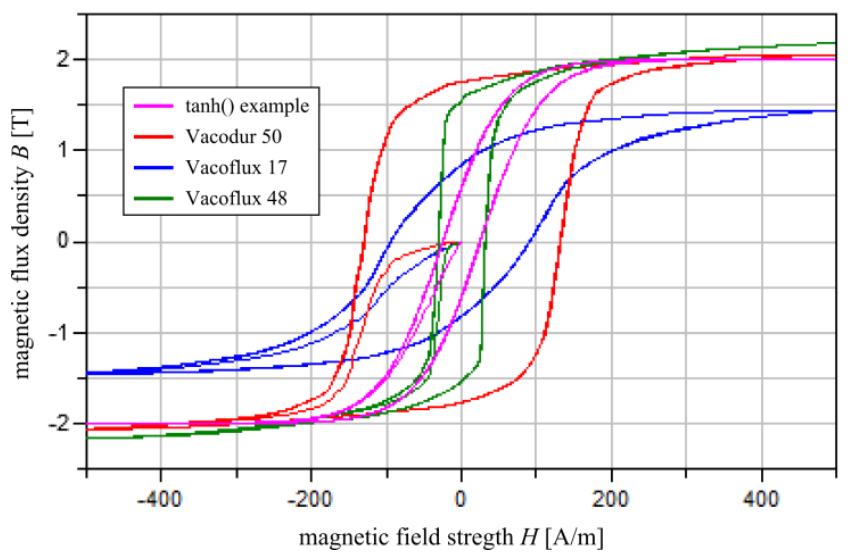

Figure 2: Exemplarily simulated limiting hysteresis loops: curve 1 described by a hyperbolic tangent function and curves 2 to 4 described by tabular $B(H)$ data extracted from [11] 


\subsection{Autotransformer as an Example}

The implemented Tellinen hysteresis models were tested with a simple electromagnetic network model of a three-phase autotransformer. A sketch of the EIshaped ferromagnetic core of the transformer with indicated corresponding network elements is shown in Figure $3 \mathrm{a}$ and the complete electromagnetic network model in Figure $3 \mathrm{~b}$.

a)
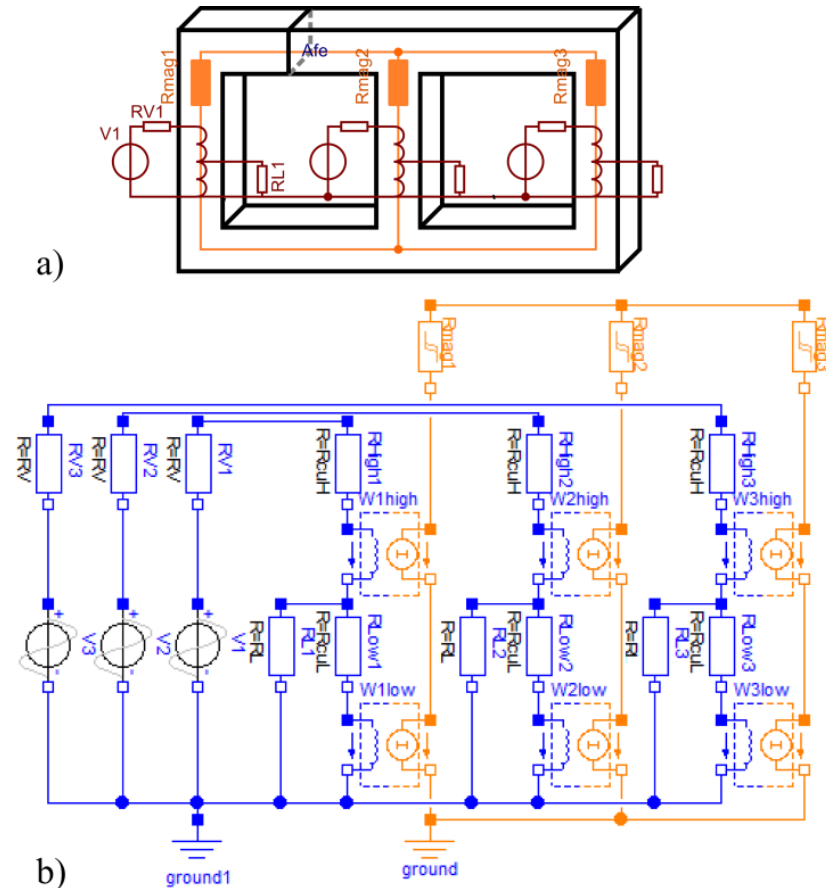

Figure 3: Sketch of a three-phase autotransformer with an EI-shaped ferromagnetic core (a) and corresponding simple electromagnetic network model with hysteresis elements representing the transformer core (b).

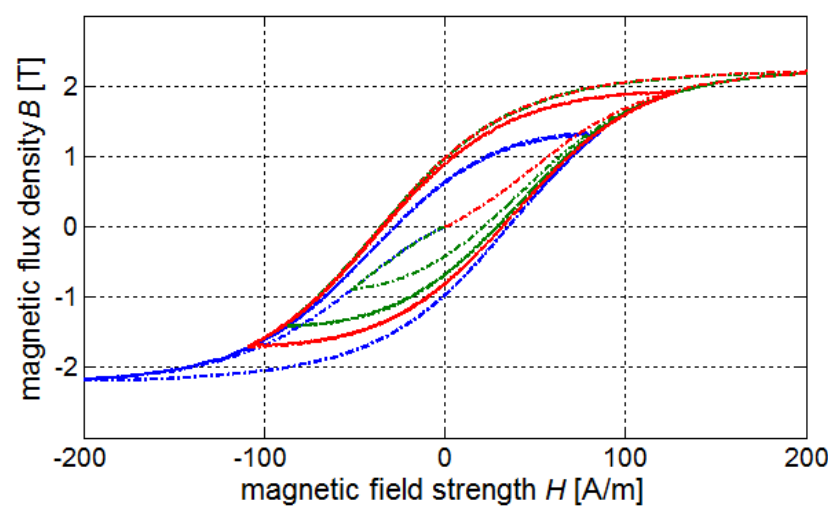

Figure 4: Simulated magnetic flux densities $B$ vs. magnetic field strength $H$ of the three hysteresis elements Rmag1 (blue), Rmag2 (red) and Rmag3 (green) representing the three transformer legs.
Transient oscillations of the magnetic flux densities in the three transformer legs after power-on are exemplarily shown in Figure 4. Selected corresponding voltages and currents are depicted in Figure 5.

a)
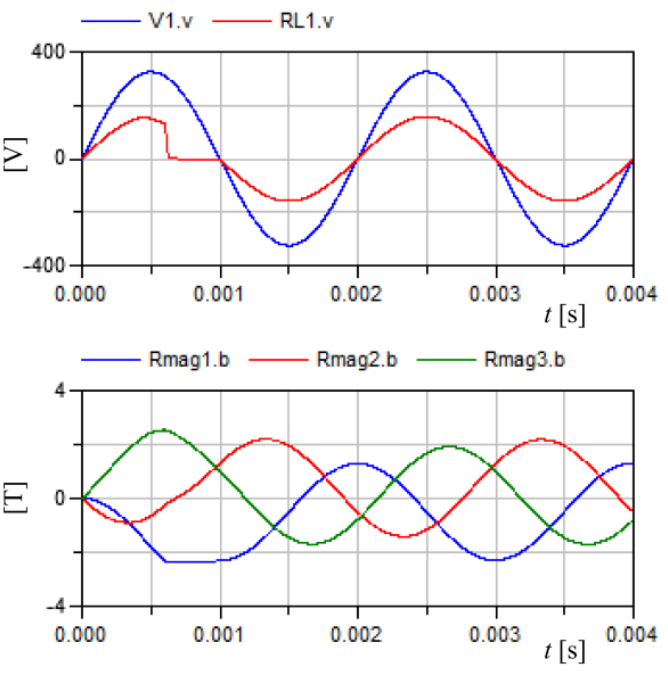

b)

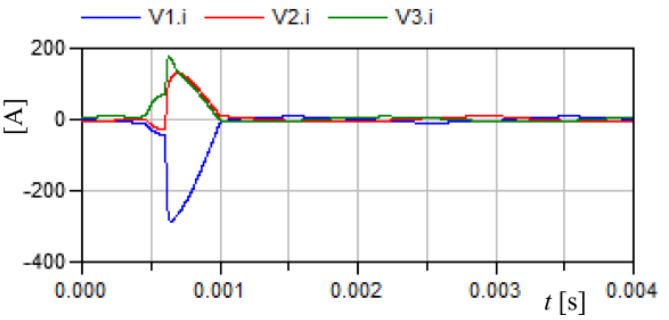

Figure 5: Results of the autotransformer simulation: source voltage V1.v and voltage drop RL1.v of load resistance (a), magnetic flux densities of the three hysteresis elements Rmag1.b to Rmag3.b (b) and source currents V1.i to V3.i.

\section{The Preisach Hysteresis Model}

\subsection{Overview on the Classical Preisach Model}

In this section a very short overview on the classical Preisach model is given. More detailed information on this model can be found e.g. in [12]. The Preisach model describes the behaviour of an output signal $j(t)$ in dependence on an input signal $h(t)$ and on its history. Here, $j(t)$ and $h(t)$ are the magnetic polarisation of a ferromagnetic material and the magnetic field strength, respectively. The model assumes an infinite set of elementary hysteresis operators $\gamma_{\alpha \beta}$. The operators' output $\gamma_{\alpha \beta} h(t)$ can only hold the polarisation values of -1 or +1 dependent on the upper and lower switching limits $\alpha$ and $\beta$, on the input signal $h(t)$ and on its history. The behaviour of $\gamma_{\alpha \beta} h(t)$ is shown in Figure 6. It is defined as 


$$
\gamma_{\alpha \beta} h(t)=\left\{\begin{array}{cl}
-1 & \text { for } h(t) \leq \beta \\
+1 & \text { for } h(t) \geq \alpha \\
\text { previous } & \text { else. }
\end{array}\right.
$$

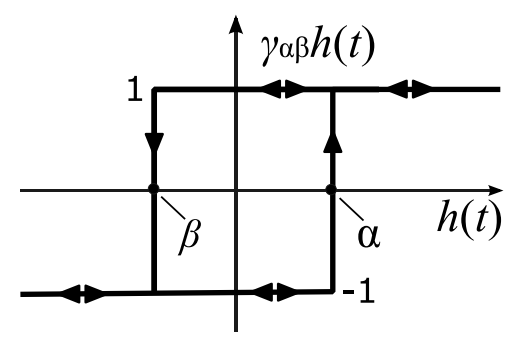

Figure 6: Elementary Preisach operator $\gamma_{\alpha \beta}$ (hysteron).

The upper switching limit of each operator is always greater than or equal to the lower limit $(\alpha \geq \beta)$. Thus, the switching limits $\alpha$ and $\beta$ span a right triangular region, often referred to as Preisach plane (Figure 7).

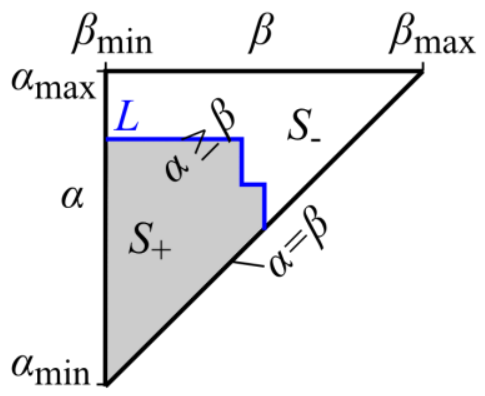

Figure 7: Preisach plane.

For each point $(\alpha, \beta)$ on this plane exactly one elementary hysteresis operator $\gamma_{\alpha \beta}$ exists with upper and lower switching limits $\alpha$ and $\beta$, respectively. The Preisach distribution function $P(\alpha, \beta)$ gives a weight to all operators in the region $\alpha \geq \beta$ and is 0 out of that region. Thus, the output polarisation $j(t)$ of the system results in

$$
j(t)=J_{S} \cdot \iint_{\alpha \geq \beta} P(\alpha, \beta) \cdot \gamma_{\alpha \beta} h(t) \mathrm{d} \alpha \mathrm{d} \beta
$$

( $J_{S}$ saturation polarisation). An exemplary Preisach distribution function is shown in Figure 8.

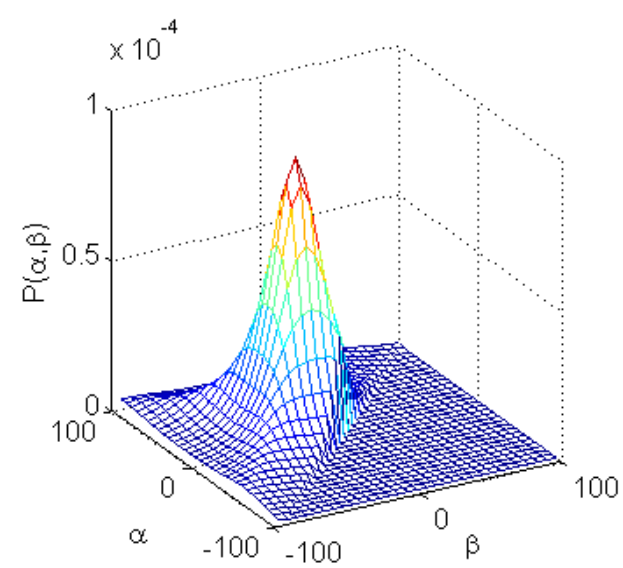

Figure 8: Exemplary Preisach distribution function $P(\alpha, \beta)$ defined over the Preisach plane $(\alpha \geq \beta)$.

The Preisach plane can be divided into two regions $S_{+}$and $S_{-}$in which all operator outputs $\gamma_{\alpha \beta} h(t)$ are in +1 and -1 state, respectively (Figure 7). Together with Eq. (5) this leads to

$j(t)=J_{S}\left(\iint_{S_{+}(t)} P(\alpha, \beta) d \alpha d \beta-\iint_{S_{-}(t)} P(\alpha, \beta) d \alpha d \beta\right)$

With the integral of $P(\alpha, \beta)$ over the region $\alpha \geq \beta$

$$
\begin{aligned}
\iint_{\alpha \geq \beta} P(\alpha, \beta) d \alpha d \beta & =\iint_{S_{+}(t)} P(\alpha, \beta) d \alpha d \beta \\
& +\iint_{S_{-}(t)} P(\alpha, \beta) d \alpha d \beta=1
\end{aligned}
$$

being equal to 1, Eq. (6) leads to

$$
j(t)=J_{S} \cdot\left(2 \cdot \iint_{S_{+}(t)} P(\alpha, \beta) d \alpha d \beta-1\right) .
$$

\subsection{Implementation in Modelica}

In general, the double integral of applied Preisach distribution functions $P(\alpha, \beta)$ cannot be expressed analytically. For that reason the numerical solution of Eq. (8) at every iteration step would be very computationally expensive. Thus, a more efficient calculation method has to be found in order to implement applicable magnetic network components in Modelica.

The evolution of both regions $S_{+}(t)$ and $S_{-}(t)$ due to a varying input signal $h(t)$ can easily be visualized in the Preisach plane (Figure 9) [12]. The hypotenuse of the Preisach plane defines the $\alpha=\beta$ line. The input signal $h(t)$ moves as a point along that line if $\alpha_{\min }<h(t)<\alpha_{\max }$. 


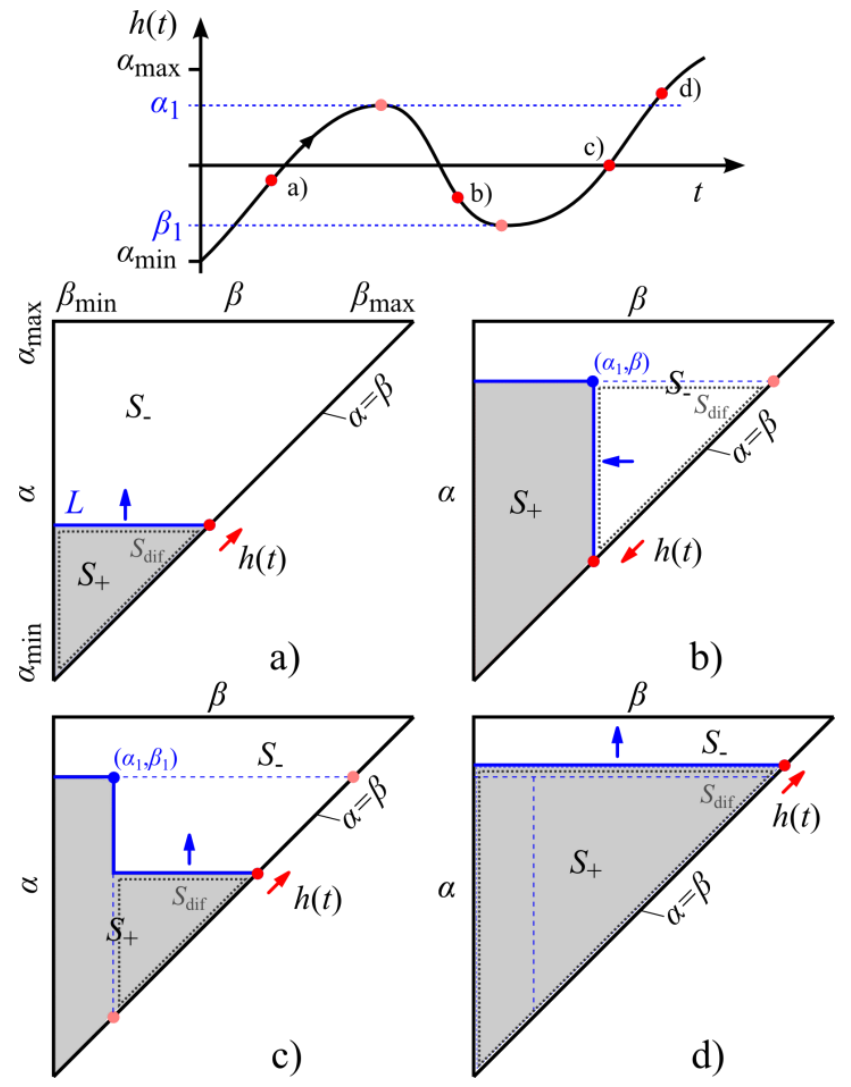

Figure 9: Geometric interpretation of the time-based evolution of the regions $S_{+}(t)$ and $S_{-}(t)$ in dependence on the input signal $h(t)$.

Starting from negative saturation (all operators are in -1 state and the whole Preisach plane is filled out by the $S$ - region) an increasing input moves a horizontal line $L$ (border between $S_{\text {- }}$ and $S_{+}$) towards the positive direction of the $\alpha$-axis, expanding the $S_{+}$region (Figure 9a). When $h(t)$ changes direction the maximum value is stored in $\alpha_{1}$ and $L$ is extended by a vertical line moving towards negative direction of the $\beta$ axis, hereby shrinking again the $S_{+}$region (Figure $9 \mathrm{~b})$. If $h(t)$ increases again, the point $\left(\alpha_{1}, \beta_{1}\right)$ is fix and $\beta_{1}$ is also stored. Dependent on the course of the input signal a corresponding number $n$ of corner points $\left(\alpha_{\mathrm{i}}, \beta_{\mathrm{i}}\right)$ must be stored. Figure $9 \mathrm{c}$ and $\mathrm{d}$ show the wiping out of stored points when $h(t)$ becomes larger than the $\alpha$ value of any stored point $\left(\alpha_{\mathrm{i}}, \beta_{\mathrm{i}}\right)$. Then this point can be deleted since it doesn't contribute any longer to the border between $S_{+}(t)$ and $S$. $(t)$. A similar event occurs when $h(t)$ becomes smaller than the last stored $\beta_{\mathrm{i}}$ value. Dependent on the number $n$ of stored points, the region $S_{+}$, over which $P(\alpha, \beta)$ must be integrated, becomes more and more complex. However, it can be shown that there is a single triangular region $S_{\text {dif }}$ (dotted triangles in Figure 9a to d) for which applies

$$
\frac{d}{d t} \iint_{S_{+}(t)} P(\alpha, \beta) d \alpha d \beta=\frac{d}{d t} \iint_{S_{d i f}(t)} P(\alpha, \beta) d \alpha d \beta .
$$

Thus, Eq. (8) and (9) lead to

$$
\frac{d j(t)}{d t}=2 \cdot J_{S} \cdot \frac{d}{d t} \iint_{S_{d i f}(t)} P(\alpha, \beta) d \alpha d \beta .
$$

$S_{\text {dif }}$ belongs to $S_{+}$for increasing $h(t)$ and to $S$ - for decreasing $h(t)$. It's hypotenuse is part of the $\alpha=\beta$ line of the Preisach plane and thus $S_{\text {dif }}$ can be written as difference of the two regions $S_{1}$ and $S_{2}$, both having their lower left vertexes at the point $\left(\alpha_{\min }, \beta_{\min }\right)$ (Figure 10). This allows to evaluate the integral of $P(\alpha, \beta)$ over the region $S_{\text {dif }}$ by two integrals with the same lower integration limits $\alpha_{\min }$ and $\beta_{\min }$ respectively:

$$
\begin{aligned}
& \iint_{S_{\text {dif }}} P(\alpha, \beta) d \alpha d \beta=\underbrace{\int_{\int_{S_{1}} P(\alpha, \beta) d \alpha d \beta}^{\beta_{2}} \int_{\alpha_{2}^{\prime}}^{\alpha_{2}^{\prime}} P(\alpha, \beta) d \alpha d \beta}_{\beta=\beta_{\min } \alpha=\alpha_{\min }} \\
& -\underbrace{\int_{\alpha=\alpha_{\min }}^{\beta_{1}} \int_{\int_{S_{2}} P(\alpha, \beta) d \alpha d \beta}^{\alpha_{2}} P(\alpha, \beta) d \alpha d \beta}_{\beta=\beta_{\min }} .
\end{aligned}
$$

With $\alpha_{\min }=\beta_{\min }=$ const., $S_{\text {dif }}$ is completely defined by the integration limits $\alpha_{2}^{\prime}, \beta_{1}^{\prime}, \beta_{2}^{\prime}$. Figure 10 shows the integration limits for increasing and decreasing $h(t)$ respectively and their variation due to a change of the input signal $h(t)$.

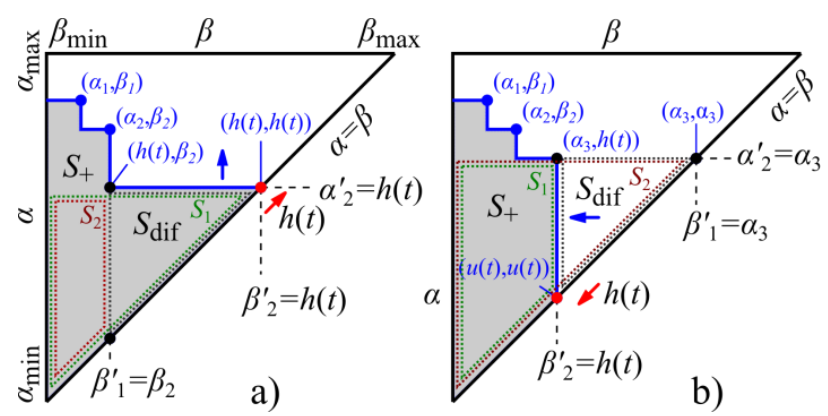

Figure 10: Integration limits $\alpha_{2}^{\prime}, \beta_{1}^{\prime}$ and $\beta_{2}^{\prime}$ of the region $S_{\text {dif }}$ for increasing (a) and decreasing (b) input signal $h(t)$.

From the integral

$$
I_{P}\left(\alpha^{\prime}, \beta^{\prime}\right)=\int_{\beta=\beta_{\min }}^{\beta^{\prime}} \int_{\alpha=\alpha_{\min }}^{\alpha^{\prime}} P(\alpha, \beta) d \alpha d \beta
$$

and Eq. (11) follows 


$$
\iint_{S_{d i f}} P(\alpha, \beta) d \alpha d \beta=I_{P}\left(\alpha_{2}^{\prime}, \beta_{2}^{\prime}\right)-I_{P}\left(\alpha_{2}^{\prime}, \beta_{1}^{\prime}\right) .
$$

With Eq. (10) and (13) one obtains

$$
\frac{d j(t)}{d t}=2 \cdot J_{S} \cdot \frac{d}{d t}\left(I_{P}\left(\alpha_{2}^{\prime}, \beta_{2}^{\prime}\right)-I_{P}\left(\alpha_{2}^{\prime}, \beta_{1}^{\prime}\right)\right)
$$

In the Preisach hysteresis model implemented in Modelica, the integral $I_{\mathrm{P}}$ of the Preisach distribution function $P(\alpha, \beta)$ is numerically computed only once at the start of a simulation run for discrete grid points and stored in a two-dimensional array $A_{\mathrm{IP}}$. All values of $I_{\mathrm{P}}$ between the grid points of $A_{\mathrm{IP}}$ can then be obtained by bilinear interpolation of adjacent $A_{\mathrm{IP}}$ values. This is an enormous reduction of the computational effort, namely from the numerical solution of the double integral of $P(\alpha, \beta)$ to two table look-ups and bilinear interpolations of $I_{\mathrm{P}}$ values in the array $A_{\mathrm{IP}}$ (see Eq. (14)). Figure 11 shows the values of $A_{\mathrm{IP}}$ for the exemplary Preisach distribution function depicted in Figure 8.

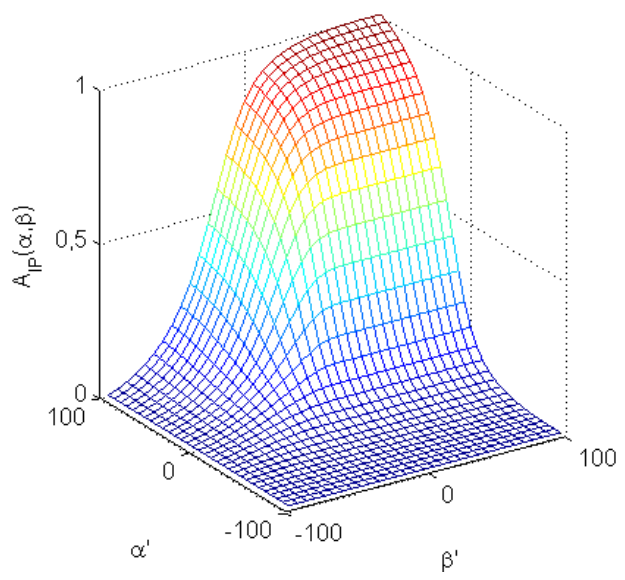

Figure 11: Array data $A_{\text {IP }}$ of the integral of the Preisach distribution function $P(\alpha, \beta)$ shown in Figure 8 .

\subsection{First Simulation Results}

A simple network model of an inductor with a closed ferromagnetic core was used for first tests of the implemented Preisach hysteresis model (Figure 12).

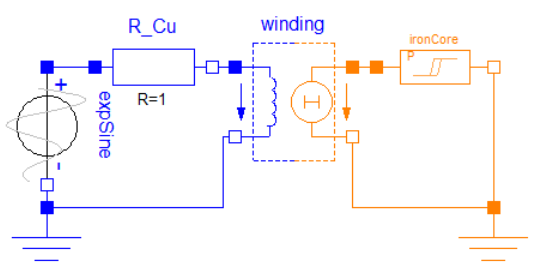

Figure 12: Simple electromagnetic model of an inductor with closed ferromagnetic core for testing of the Preisach hysteresis model.
Simulation results, especially the simulated $B(H)$ hysteresis of the iron core, are shown in Figure 13. The increasing exponential sine voltage causes growing hysteresis loops. The resulting $B(H)$ loops are not centered around the origin, because the flux density $B$ of this simulation starts for $H=0 \mathrm{~A} / \mathrm{m}$ at negative remanence.
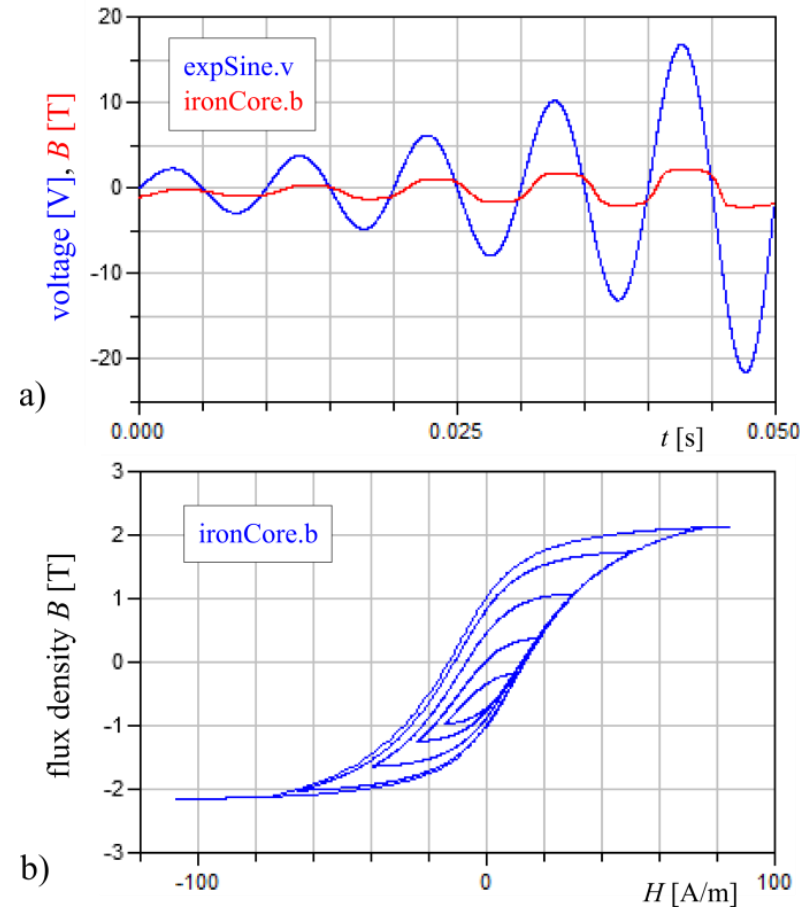

Figure 13: Simulation results of the inductor model: source voltage expSine.v and flux density ironCore.b in the core (a) and $B(H)$ plot of the growing hysteresis loops in the iron core (b).

\section{Model Comparison}

To show the different behaviour between the classical Preisach and the Tellinen hysteresis model two simulations were carried out. An identical magnetic field strength $H(t)$ was applied to the input of both hysteresis elements, which were configured to have equal limiting hysteresis loops. The models output characteristics $B(H)$ were then plotted together in one diagram. In the first simulation a decreasing exponential sine wave was used as input signal. The corresponding simulation results are shown in Figure 14. Only small differences in the models output are obvious. The different behaviour can be seen more clearly in the results of the second simulation, in which a slightly more complex input signal of two superposed sine waves of different amplitude and frequency (Figure 15a) was applied. The $B(H)$ characteristics in Figure 15b show the deviation between both models, especially in the region of the minor 
loops. In contrast to the Tellinen model, the minor loops of the classical Preisach model are closed.

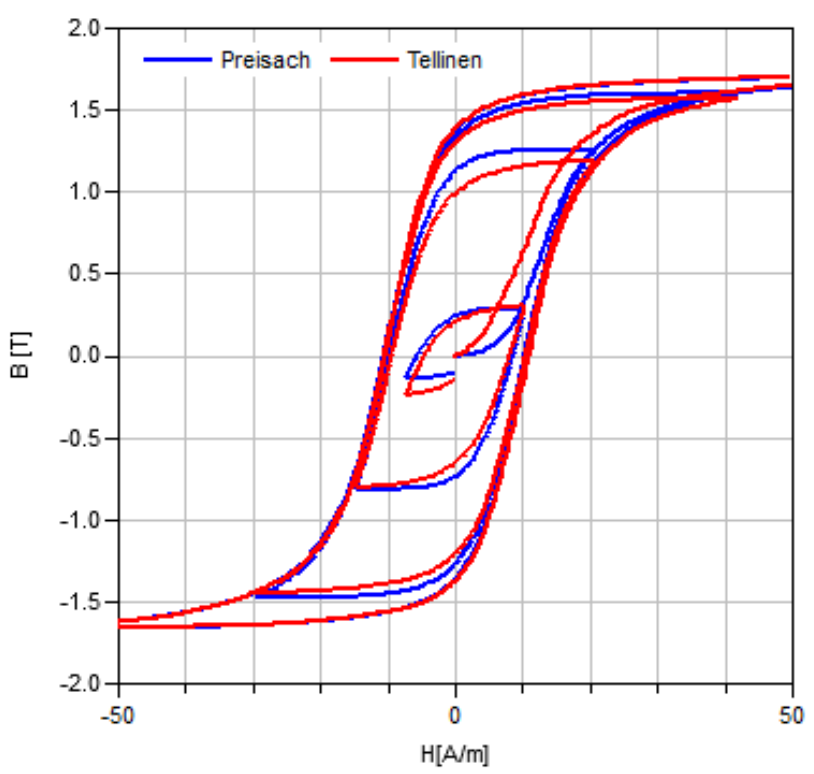

Figure 14: $B(H)$ characteristics of the Preisach and the Tellinen hysteresis model for a decreasing exponential sine wave input signal $H(t)$.

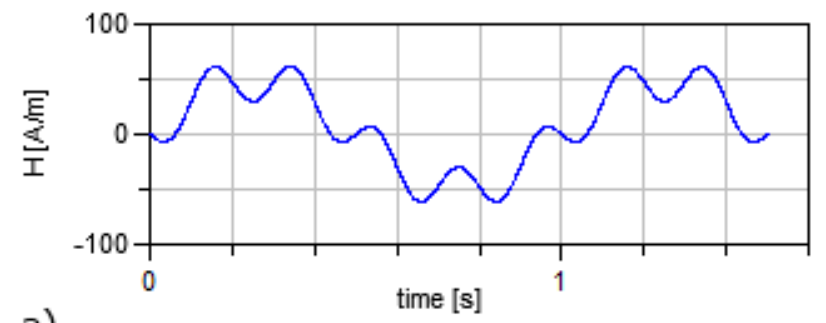

a)

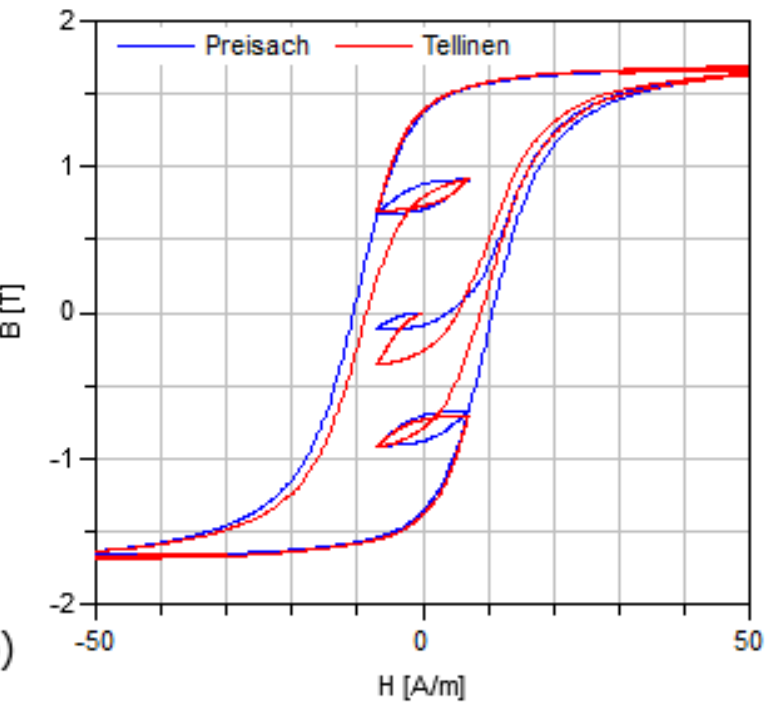

Figure 15: Output of the Preisach and Tellinen model (b) for the identical input signal (a).
Due to the significantly higher computational effort for the Preisach model the network simulation with the Tellinen model performs a lot faster. Dependent on the fineness of the mesh of the discretised Preisach integral, a simulation with one Preisach hysteresis element takes about 3 to 8 times as long as a similar simulation with a Tellinen hysteresis network element.

\section{Summary and Outlook}

Two different magntic hysteresis models have been implemented in Modelica: the simple but efficient model developed by Tellinen and the more accurate but complex Preisach model. For latter model, a particular simple and efficient Modelica implementation was derived, hereby reducing the effort for numerical calculation of a double integral over portions of the Preisach plane to two bilinear interpolations in a table.

Utilisation of the Tellinen model together with components of the Modelica.Magnetic.FluxTubes library was exemplarily shown with transient simulation of a three-phase autotransformer.

With further work, the developed hysteresis models will be optimised and tested. Estimation of hysteresis losses from simulated hysteretic behaviour will be implemented. Those simulated iron losses will be provided to a conditional heat port and thus can be input to subsequent thermal simulations, e.g. with models built from Modelica. Thermal. HeatTransfer. Further improvements of the developed hysteresis models will focus on proper initialisation as well as on numerical stability and computational efficiency. If reasonable, the well-known JilesAtherton model of magnetic hysteresis will be also implemented. All implemented hysteresis models will be compared with regard to behaviour, accuracy and computation time.

For model validation, measurements of the magnetic properties of selected magnetically soft materials according to EN 60404 are planned. A measurement setup utilising a highly accurate electronic fluxmeter is currently realised. With data obtained from these measurements, the materials sublibrary of Modelica.Magnetic.FluxTubes will be extended and improved. For the Preisach hysteresis model a corresponding parameter identification needs also to be developed for fitting the model behaviour to literature or measured hysteresis data. 


\section{Acknowledgement}

The authors would like to thank the Clean Sky Joint Technology Initiative for funding of the presented work within Project No. 296369 MoMoLib "Modelica Model Library Development for Media, Magnetic Systems and Wavelets".

\section{References}

[1] Modelica Association, Modelica Standard Library, https://www.modelica.org/libraries/Modelica (May 11, 2012).

[2] T. Bödrich and T. Roschke, A Magnetic Library for Modelica, in Proc. of the 4th International Modelica Conference, 2005, pp. 559565.

[3] T. Bödrich, Electromagnetic Actuator Modelling with the Extended Modelica Magnetic Library, Proc. of 6th Int. Modelica Conf., Bielefeld, Germany, March 3-4, pp. 221-227, 2008.

[4] H. Roters, Electromagnetic Devices. New York: John Wiley \& Sons, 1941.

[5] C. Steinmetz, Hysteresis loss, Electrician 26, p. $261 \mathrm{ff} ., 1891$.

[6] T. Roschke, Entwurf geregelter elektromagnetischer Antriebe für Luftschütze, ser. FortschrittBerichte VDI. VDI Verl., 2000.

[7] D. Ribbenfjärd, Electromagnetic Modelling Including the Electromagnetic Core, Ph.D. dissertation, KTH Royal Institute of Technology, Stockholm, 2010.

[8] F. Preisach, Über die magnetische Nachwirkung, Zeitschrift für Physik A Hadrons and Nuclei, vol. 94, pp. 277-302, 1935.

[9] D. Jiles and D. Atherton, Theory of Ferromagnetic Hysteresis, Journal of Magnetism and Magnetic Materials, vol. 61, no. 1-2, pp. 48 60, 1986.

[10] J. Tellinen, A Simple Scalar Model for Magnetic Hysteresis, IEEE Transactions on Magnetics, vol. 24, no. 4, pp. 2200 - 2206, July 1998.

[11] Soft Magnetic Cobalt-Iron-Alloys, Vacuumschmelze GmbH, 2001, http://www.vacuumschmelze.com/fileadmin/docroot/medialib/documents/broschue-ren/htbrosch/Pht004_e.pdf (05.21.2012).

[12] I. Mayergoyz, Mathematical Models of Hysteresis and their Application. Elsevier, 2003. 